\title{
Supplementing Regular Training With Short-Duration Sprint-Agility Training Leads to a Substantial INCREASE IN RePEATEd Sprint-Agility Performance With National Level Badminton Players
}

\author{
Benjamin M. Walklate, ${ }^{\mathbf{1}}$ Brendan J. O'Brien, ${ }^{\mathbf{1}}$ Carl D. Paton, ${ }^{\mathbf{2}}$ and Warren Young ${ }^{\mathbf{1}}$ \\ ${ }^{1}$ School of Human Movement and Sport Sciences, University of Ballarat, Victoria, Australia 3353; and ${ }^{2}$ Eastern Institute of \\ Technology, Napier, New Zealand 4142
}

\begin{abstract}
Walklate, BM, O'Brien, BJ, Paton, CD, and Young, W. Supplementing regular training with short-duration sprint-agility training leads to a substantial increase in repeated sprint-agility performance with national level badminton players. J Strength Cond Res. 23(5): 1477-1481, 2009-Repeated-agility sprint ability is an important performance characteristic of badminton players. However, it is unclear whether regular badminton training is sufficient to improve repeated-agility sprint ability or whether supplementary training is required. Therefore, our aim was to investigate whether supplementing regular group training with short sessions of badminton-specific agility-sprint training conferred any greater changes in performance than regular training alone. Twelve national level badminton players completed a set of performance tests in the week before and after a 4-week training period. Performance tests consisted of 10- and 20meter sprints, a multistage fitness test, a 300-meter shuttle run, and a novel badminton sprint protocol. After pretesting, pairmatched participants were randomly assigned into regular or supplementary training groups. Both groups undertook regular national squad training consisting of 42 -hour sessions per week. In addition, the supplementary group completed a highintensity sprint-training regime consisting of 7 to 15 repeats of badminton-specific sprints twice per week. Relative to control, the supplementary training group reported improvements (mean $\pm 90 \%$ confidence limits) in the 300-meter shuttle run $(2.4 \% \pm 2.7 \%)$ and badminton sprint protocol (3.6\% $\pm 2.6 \%)$. However, there were no substantial difference in either the 10meter $(0.3 \% \pm 2.1 \%)$ or 20 -meter $(0.6 \% \pm 1.8 \%)$ sprint or the multistage fitness test $(0.0 \% \pm 2.7 \%)$. Supplementing
\end{abstract}

Address correspondence to Brendan J. O’Brien, b.obrien@ballarat.edu.au 23(5)/1477 1481 regular training with sessions of short-duration sprint training appears to lead to worthwhile increases in repeated-agility sprint performance with national level badminton players.

KEY WoRDS raquet sports, intermittent run, speed work

\section{INTRODUCTION}

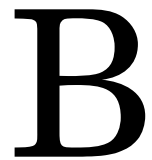
adminton is a highly competitive dynamic sport requiring great demands on the cardiorespiratory and muscular systems. Heart rates have been reported to approach $100 \%$ of maximum heart rate and average $169 \pm 9$ b.min ${ }^{1}$ during competition (7). Analysis of blood lactate concentrations during competition show values may reach 3 to 6 mmol.L ${ }^{1}$ (12). Motion analysis of a typical badminton match reveals that there are many changes in direction, and points range in duration between 1 and 40 seconds, with the average point lasting 6.8 seconds and the rest period between points being only 5 to 10 seconds (3). Consequently, it appears that badminton players should maximize their repeated agility sprint capabilities to enhance their on court success. However, there is little information on whether repeated agility sprint ability (RASA) for badminton players is improved through conventional training or whether supplementary agility sprint training will result in greater performance gains. Several researchers have demonstrated that isolated repeated sprint training has a positive effect on lactate tolerance, maximal aerobic power, muscle buffer capability, and the rate of phosphate creatine resynthesis, resulting in superior repeated sprint performance $(15,9)$. However it is not possible to distinguish whether supplementary training confers any further benefit. It is possible that regular badminton squad training may be insuf ficient to induce improvements in repeated sprint ability. Spencer et al. (16) demonstrated that regular field hockey training improved absolute total work by approximately $2 \%$ during $5 \times 6$ second maximal sprints. However, despite the positive physiologic changes observed with regular training 
by Spencer et al. (16) and isolated repeated sprint training, it is still unclear whether regular badminton team training will improve repeated sprint ability or whether a specific training protocol that simulates the movement patterns in a typical point in badminton will result in greater performance. There fore, the primary aim of this study was to investigate whether specific supplementary repeated sprint training confers any advantage in repeated sprint fitness over and above tradi tional training. Furthermore, a second objective was to determine the reliability of a test specifically developed to assess repeated sprint fitness in badminton players.

\section{Methods}

\section{Experimental Approach to the Problem}

In this investigation, 12 (6 males, 6 females) elite badminton players were randomly assigned into 2 treatments: a regular training group (REG) and a group that supplemented regular training with prior repeated sprint training (SUP). A test of repeated sprint ability, 10 and 20 meter sprint times, a multi stage fitness test, and a 300 meter shuttle test were performed before and after completion of 4 weeks of training. In REG, participants trained 4 times per week for 4 weeks, and each session lasted approximately 2 hours. In SUP, participants completed a high intensity sprint training regime twice a week in addition to regular training. The sprint training was performed immediately before the regular training sessions.

\section{Subjects}

Twelve members of the Australian national elite badminton squad volunteered to participate in this study in the precom petition training phase. The participants had the following characteristics: age $=19 \pm 1.8$ years; height $=168 \pm 6 \mathrm{~cm}$; mass $=70 \pm 6 \mathrm{~kg}$. The athletes provided written informed consent, and the study was approved by the human research ethics committee of the University of Ballarat.

\section{Procedures}

Twenty four hours before reporting to each laboratory session, each participant was requested to eat approximately 8 to $10 \mathrm{~g}$ of carbohydrate per kilogram of body weight, sleep a minimum of 7 hours, and drink adequate fluid to ensure a hydrated state. Upon reporting to the national badminton training center in Melbourne (ambient temperature $1822^{\circ} \mathrm{C}$ ), participants initially performed a warm up protocol con sisting of 5 minutes of light jogging and stretching of the leg muscles.

On completion of the warm up, the participants completed a badminton specific test of RASA. The repeated agility sprint test required the participant to perform 1020 second agility sprints on a badminton court, with 10 seconds of passive recovery between efforts. Reference cones were placed on the agility course devised to delineate reference points. The test measured the number of reference points placed at set intervals around the standard badminton court the player passed in each 20 second agility sprint. The agility
TABLE 1. Supplementary short repeated-sprint training program, revealing the work and rest times and the repetitions (sets) completed each session.

\begin{tabular}{lccr}
\hline Week & Training session & Work:rest (s) & Sets \\
\hline 1 & Pretesting & & \\
2 & 1 & $20: 10$ & 7 \\
& 2 & $20: 10$ & 9 \\
3 & 3 & $20: 10$ & 9 \\
& 4 & $20: 10$ & 11 \\
4 & 5 & $20: 10$ & 11 \\
& 6 & $20: 10$ & 13 \\
5 & 7 & $20: 10$ & 13 \\
& 8 & & \\
6 & Post-testing
\end{tabular}

sprints required the participant to sprint from the forehand rear of a badminton court to the forehand front corner then diagonally back to the backhand rear corner, then to the front backhand corner, and finally back to the forehand rear corner of the badminton court to complete each circuit. From the initial starting position, 4 reference points were placed $1.35 \mathrm{~m}$ apart along the forehand rear to forehand front of the court, 4 reference points were placed $1.87 \mathrm{~m}$ apart along the forehand front to the backhand rear of the court, 4 reference points were placed $1.35 \mathrm{~m}$ apart along the backhand rear to backhand front, and 4 reference points were placed $1.87 \mathrm{~m}$ apart on the forehand rear corner of the badminton courts. When participants finished between 2 reference points, the reference point last reached was used to record the distance completed. A fatigue index score was determined as the

TABLE 2. Characteristics of performance of athletes in control and supplementary training groups.*

\begin{tabular}{|c|c|c|}
\hline & $\begin{array}{l}\text { Control } \\
(n=6)\end{array}$ & $\begin{array}{c}\text { Experimental } \\
\quad(n=6)\end{array}$ \\
\hline 10-m sprint time (s) & $1.98 \pm 0.07$ & $1.94 \pm 0.18$ \\
\hline 20-m sprint time (s) & $3.44 \pm 0.14$ & $3.35 \pm 0.30$ \\
\hline 300-m time (s) & $76.3 \pm 6.0$ & $73.3 \pm 3.4$ \\
\hline $\begin{array}{l}\text { Badminton shuttle } \\
\text { distance }(\mathrm{m})\end{array}$ & $351 \pm 31$ & $365 \pm 10$ \\
\hline $\begin{array}{l}\text { Badminton shuttle } \\
\text { decrement (\%) }\end{array}$ & $23.9 \pm 8.0$ & $25.2 \pm 8.1$ \\
\hline $\begin{array}{l}20-m \text { maximal shuttle } \\
\text { run test (stage) }\end{array}$ & $10.4 \pm 2.2$ & $12.0 \pm 0.6$ \\
\hline
\end{tabular}

${ }^{*}$ Data are mean \pm between-subject $S D$. 
TABLE 3. Changes in performance measures in control and experimental training groups.

\begin{tabular}{lcccc}
\hline & \multicolumn{3}{c}{ Change in measure (\%) } \\
\cline { 2 - 4 } & $\begin{array}{c}\text { Control, } \\
\text { mean } \pm \text { SD }\end{array}$ & $\begin{array}{c}\text { Experimental, } \\
\text { mean } \pm S D\end{array}$ & $\begin{array}{c}\text { Difference, } \\
\pm 90 \% \text { CL }^{*}\end{array}$ & Cohen effect size \\
\hline 10-m sprint time & $0.6 \pm 1.6$ & $0.3 \pm 2.2$ & $0.3 ; \pm 2.1$ & 0.04 \\
20-m sprint time & $0.4 \pm 1.6$ & $0.2 \pm 1.7$ & $0.6 ; \pm 1.8$ & 0.10 \\
300-m time & $2.6 \pm 0.9$ & $5.0 \pm 3.2$ & $2.4 ; \pm 2.7$ & 0.36 \\
Badminton shuttle distance & $1.4 \pm 1.2$ & $5.0 \pm 3.1$ & $3.6 ; \pm 2.6$ & 0.55 \\
Badminton shuttle decrement & $2.2 \pm 3.5$ & $7.5 \pm 3.5$ & $5.3 \pm 3.5$ & 0.68 \\
20-m maximal shuttle run test & $1.2 \pm 2.0$ & $1.3 \pm 2.9$ & $0.0 ; \pm 2.7$ & 0.00 \\
\hline
\end{tabular}

${ }^{*} \pm 90 \% \mathrm{CL}=$ add and subtract this number to or from the mean effect to obtain the $90 \%$ confidence limits $(\mathrm{CL})$ or the true difference.

percentage decrement in distance covered calculated be tween the 1st sprint and 10th sprint.

The following day at the same venue, 10 and 20 meter sprint times were determined using dual beam electronic timing lights (Swift, Lismore, Australia). Participants were instructed to adopt a stationary standing start position with the leading toe just before the $0 \mathrm{~m}$ mark. Each participant was instructed to exert a maximal effort, and the fastest result recorded from three maximal sprint efforts was used for analysis. Upon completion of the speed test, athletes rested for 30 minutes before undertaking a maximal effort 300 meter shuttle run. Using the same 20 meter course used in the sprint test, the participants sprinted the $20 \mathrm{~m}$ distance back and forth continuously 15 times $(300 \mathrm{~m})$ and were instructed to run in a straight line in the quickest possible time. Time to complete the $300 \mathrm{~m}$ was recorded to the nearest second. Forty eight hours later, athletes completed a 20 meter multi stage shuttle run test (14) for assessment of peak running velocity and predicted maximum oxygen consumption.

After pretesting, pair matched participants were randomly assigned into regular or supplementary training groups. Both groups undertook regular national badminton squad training consisting of approximately 42 hour sessions per week. In addition, the supplementary group completed a progressive high intensity sprint training regime immediately before normal scheduled training. The training regime required participants to complete repeated 20 second sprints with 10 seconds of recovery between sprints using the same circuit adopted in the badminton simulation test. The number of sprints was gradually increased from 7 to 15 over the 4 week training period. The rate of progression is depicted in Table 1.

Upon completion of the 8 training sessions, participants completed the post tests in the same order, at the same time of day, with the same recovery duration between each test. In addition to the post tests, the repeated sprint test was repeated on a further occasion 48 hours after the multistage fitness test to establish the reliability (test retest error) of the test.

\section{Statistical Analyses}

Simple group statistics are shown as means \pm between subject $S D$. Performance data are presented and analyzed as mean $\pm S D$ for repeat (pre post) trials under control and supplementary training conditions. The mean effects of sup plementary training and their $90 \%$ confidence limits were estimated with a spreadsheet (10) by way of the unequal variances $t$ statistic computed for change scores between pre tests and post tests. Subjects' change scores were expressed as a percentage of baselines by way of analysis of $\log$ transformed values to reduce any bias arising from nonuniformity of error in the data. Magnitude of the change scores was also determined and interpreted using the effect size statistic (change in mean divided by the between subject $S D$ in the pretest) according to the following thresholds as defined by Cohen (4): less than $0.2=$ trivial, 0.2 to $0.6=$ small, 0.6 to $1.2=$ moderate, 1.2 to $2.0=$ large, greater than $2.0=$ very large. Errors of measurement for the badminton sprint test were expressed as the coefficients of variation $(\mathrm{CV})$ based on repeated trial data and were also estimated with the spreadsheet.

\section{Results}

The baseline characteristics and performance for each training group are depicted in Table 2. There were in substantial differences in performance in both 10 meter and 20 meter sprint time and multistage fitness test between the training groups (Table 3). However, there were small to moderate improvements (as defined by effect size) in both the badminton repeated sprint test and the 300 meter shuttle test in the SUP group relative to the REG groups.

The CV for the sport specific badminton test was $0.9 \%$ (90\% confidence intervals $0.61 .8 \%$ ) and $13.0 \%$ (9.5 20.8) for the total distance covered and the rate of fatigue, respectively.

\section{Discussion}

The aim of this study was to determine whether the addi tion of 4 weeks of supplementary badminton specific 
repeated sprint training (SUP) before regular badminton training (REG) facilitated a greater change in an athlete's RASA. Our main finding was that SUP substantially improved RASA as determined by a greater accumulation of reference points (approximately 5\%) and attenuated the rate of fatigue (approximately 5\%) in the performance test after training. REG did not alter the indicators of RASA over time.

Our findings indicate that a substantial training effect in RASA can occur with only 5 minutes of additional badminton specific repeated sprint training performed twice per week for a 4 week period. Therefore, 26 minutes of 20 second repeated sprint training over 4 weeks appears to be a sufficient training stimulus to induce worthwhile improve ments in badminton repeated sprint ability. A relatively small dose of exercise required to elicit improvements in repeated sprint performance has previously been reported by Burgomaster et al. (2). Burgomaster et al. demonstrated that 15 minutes of short duration sprint training ( 47 allout efforts of $30 \mathrm{~s}$ ) performed for 6 sessions is sufficient to double endurance capacity during constant rate cycling at $80 \%$ of $\mathrm{VO}_{2 \text { max }}$ and peak power output during repeat sprints. Further information on the minimal and optimal doses of training required to improve RASA is required, but presently it would appear that the training time required by the athlete to improve RASA should not be constraining.

The adaptations that facilitate an improvement in RASA include physiologic changes that improve the metabolic capacities and power output of the athlete and may be consequential to several possible changes, including an eleva tion in the glycolytic enzymes phosphofructokinase and hexokinase (11), greater intramuscular glycogen reserves (2), enhancement of the maximal rate of oxygen uptake, and an increase proportion of type 2 muscle fibers (5). Our study attempted to determine the mechanism for an enhancement of RASA by measuring changes in maximal oxygen uptake by way of the multistage fitness test, improved peak force production by way of 10 and 20 meter sprint efforts, and anaerobic capacity by way of the 300 meter shuttle test. Our results indicated that neither REG nor SUP training created sufficient stimulus to improve the maximal oxygen uptake, and consequently it is unlikely that an enhancement of the body's ability to aerobically produce adenosine triphosphate (ATP) was a significant contributor to improved performance in the RASA test. Peak speed was found to be unchanged after engagement in both SUP and REG. However, perfor mance in the 300 meter shuttle test improved substantially more in SUP, suggesting that the additional training increased the participant's capacity to produce ATP anaerobically. Performance on the 300 meter shuttle test correlates strongly with the maximal accumulated oxygen deficit (13), a measure used to assess an athlete's anaerobic capacity. Simultaneous improvements in repeated sprint ability and the 300 meter shuttle distance covered were expected because the distances covered during the test were similar.
An alternative mechanism to an increase in the metabolic capacity and peak power output is an increase in fatigue resistance. An increase in muscle buffer capability is a poten tial mechanism that facilitates greater RASA by minimizing muscle $\mathrm{pH}$ changes induced by exercise and in turn enables the athlete to sustain force production for longer (6). Muscle buffer capability has been shown to increase with 5 weeks of repeated sprint training (1). In addition, it is possible that the improvement in RASA could be attributed to an enhance ment of the athlete's mental/psychological tolerance during the RASA test.

The second objective of the present study was to report the retest error (reliability) of the test. It is essential that all tests of exercise performance report a retest error to distinguish genuine training effects from artifacts of the data. Our data suggest that the badminton RASA test we developed was reliable enough to detect small changes in RASA if total accumulated reference points were used. However, the percent decrement method of determining fatigue during the test is less reliable, yielding a large CV of approximately $13 \%$. Our results concur with the findings of other studies that have demonstrated good reliability for total work performed but poor reliability for measures of fatigue. Spencer et al. (17) reported a CV of approximately $1 \%$ for total sprint time of $6 \times$ $30 \mathrm{~m}$ sprints departing every 24 seconds and approximately $15 \%$ when percent decrement was used to assess RSA. Similarly, Fitzsimmons et al. (8) reported a CV of approx imately $1 \%$ for $6 \times 40 \mathrm{~m}$ sprints departing every 30 seconds, but the $\mathrm{CV}$ increased to $18.5 \%$ when RASA was assessed by percent decrement. Consequently, percent decrement may not be a reliable method of measuring fatigue during the RASA because the CV is high and will lack the sensitivity to detect likely RASA changes attributable to training. A more reliable method of assessing RASA is therefore measurement of total work done, which, in effect, are the total reference points accumulated as represented in our study. In con clusion, we report that 7 advancing to 15 sets of 20 second sprints interspersed with 10 seconds of rest performed twice a week for 4 weeks will improve RASA. The mechanisms that occur from SUP that enhance RASA are not con sequential to improvements in aerobic power and maximal speed. The greater improvement in RASA is more likely attributable to an enhanced anaerobic capacity reflected by a similar percentage improvement in the 300 meter shuttle run in the RASA. The RASA test developed has low retest error if total reference points accumulated are used to effectively gauge training changes but has a high error if percent decrement is used.

\section{Practical Applications}

The practical implications of this study are that supplement ing regular badminton training with sessions of high intensity sprint training may confer additional improvements in RASA beyond regular training alone and should be considered beneficial as a potential performance enhancing strategy. 
Furthermore, RASA is assessed most appropriately by total accumulated reference points (total work done) in a sportspecific badminton test.

\section{ACKnowledgments}

The authors acknowledge the technical assistance provided by Andrew Perks. The study was funded by the University of Ballarat. The results of the study do not constitute endorsement by the NSCA.

\section{REFERENCES}

1. Bishop, D, Edge, J, and Goodman, C. Muscle buffer capacity and aerobic fitness are associated with repeated-sprint ability in women. Eur J Appl Physiol 92: 540-547, 2004.

2. Burgomaster, KA, Hughes, SC, Heigenhauser, GJ, Bradwell, SN, and Gibala, MJ. Six sessions of sprint interval training increases muscles oxidative potential and cycle endurance capacity in humans. $J$ Appl Physiol 98: 1985-1990, 2005.

3. Cabello-Manrique, D and Gonzalez-Badillo, JJ. Analysis of the characteristics of competitive badminton. BrJ Sports Med 37: 62-66, 2003.

4. Cohen, J. Statistical power analysis for the behavioral sciences (2nd ed.). Mahwah, NJ: Lawrence Erlbaum, 1988.

5. Dawson, B, Fitzsimons, B, Green, S, Goodman, C, Carey, M, and Cole, K. Changes in performance, muscle metabolites, enzymes and fibre types after short sprint training. Eur J Appl Physiol 78: 163-169, 1998.

6. Edge J, Bishop, D, Goodman, C, and Dawson, B. Effects of high- and moderate-intensity training on metabolism and repeated sprints. Med Sci Sport Exerc 37: 1975-1982, 2005.
7. Faccini, $\mathrm{P}$ and Dal Monte, A. Physiologic demands of badminton match play. Am J Sports Med 24: 564-566, 1996.

8. Fitzsimmons, M, Dawson, B, Ward, D, and Wilkinson, A. Cycling and running tests of repeated sprint ability. Aust J Sci Med Sport 25: 82-87, 1993

9. Glaister, M. Multiple sprint work: physiological responses, mechanisms of fatigue and the influence of aerobic fitness. Sports Med 35: 757-777, 2005.

10. Hopkins, WG. A spreadsheet for analysis of straightforward controlled trials. Sportscience. Available at: sportsci.org/jour/03/ wghtrials.htm. Accessed in 2007.

11. Macdougall, J, Hicks, A, Macdonald, J, Mckelvie, R, Green, H, and Smith, K. Muscle performance and enzymatic adaptations to sprint interval training. J Appl Physiol 84: 2138-2142, 1998.

12. Majumdar, P, Khanna, GL, Malik, V, Sachdeva, S, Arif, M, and Mandal, M. Physiological analysis to quantify training load in badminton. Br J Sports Med 31: 342-345, 1997,

13. Moore, A and Murphy, A. Development of an anaerobic capacity test for field sport athletes. J Sci Med Sport 6: 275-284, 2003.

14. Paliczka, VJ, Nichols, AK, and Boreham, CA. A multi-stage shuttle run as a predictor of running performance and maximal oxygen uptake in adults. Br J Sports Med 21: 163-165, 1987.

15. Spencer, M, Bishop, D, Dawson, B, and Goodman, C. Physiological and Metabolic Responses of Repeated-Sprint Activities. J Sports Med 35: 1025-1044, 2005.

16. Spencer, M, Bishop, D, and Lawrrence, S. Longitudinal assessment of the effects of field-hockey training on repeated sprint ability. $J$ Sci Med Sport 7: 323-334, 2004.

17. Spencer, M, Fitzsimons, M, Dawson, B, Bishop, D, and Goodman, C. Reliability of a repeated-sprint test for field-hockey. J Sci Med Sport 9: 181-184, 2006. 